\title{
Multimodal Gaze Interaction for Creative Design
}

\author{
Chris Creed \\ DMT Lab, Birmingham City \\ University, UK \\ chris.creed@bcu.ac.uk
}

\author{
Maite Frutos-Pascual \\ DMT Lab, Birmingham City \\ University, UK \\ maite.frutos@bcu.ac.uk
}

\author{
Ian Williams \\ DMT Lab, Birmingham City \\ University, UK \\ ian.williams@bcu.ac.uk
}

\begin{abstract}
We present a new application ("Sakura") that enables people with physical impairments to produce creative visual design work using a multimodal gaze approach. The system integrates multiple features tailored for gaze interaction including the selection of design artefacts via a novel grid approach, control methods for manipulating canvas objects, creative typography, a new color selection approach, and a customizable guide technique facilitating the alignment of design elements. A user evaluation $(N=24)$ found that nondisabled users were able to utilize the application to complete common design activities and that they rated the system positively in terms of usability. A follow-up study with physically impaired participants $(N=6)$ demonstrated they were able to control the system when working towards a website design, rating the application as having a good level of usability. Our research highlights new directions in making creative activities more accessible for people with physical impairments.
\end{abstract}

\section{Author Keywords}

Eye gaze tracking; gaze interaction; interface design; eye gaze design.

\section{CSS Concepts}

- Human-centered computing $\rightarrow$ Systems and tools for interaction design.

\section{INTRODUCTION}

Eye gaze interaction has received significant attention from researchers over the past three decades [37]. A strong focus of this work has been around investigating novel eye typing approaches [40], as well as exploring unique interaction possibilities and issues associated with gaze input (e.g. the Midas touch issue [25], the selection of small targets [1, 4, $6,15,31,45,62,63]$, and scrolling documents [7, 31, 53, $56,68,70])$. However, whilst substantial progress has been made in these areas, there has been a lack of research to date on the use of gaze interaction for supporting more visual creative work. The ability to efficiently perform different design and graphical tasks (e.g. the manipulation of images) via gaze tracking is essential for some key user

Permission to make digital or hard copies of all or part of this work for personal or classroom use is granted without fee provided that copies are not made or distributed for profit or commercial advantage and that copies bear this notice and the full citation on the first page. Copyrights for components of this work owned by others than the author(s) must be honored. Abstracting with credit is permitted. To copy otherwise, or republish, to post on servers or to redistribute to lists, requires prior specific permission and/or a fee. Request permissions from Permissions@acm.org. CHI 2020, April 25-30, 2020, Honolulu, HI, USA.

(C) 2020 Copyright is held by the owner/author(s). Publication rights licensed to ACM. ACM ISBN 978-1-4503-6708-0/20/04...\$15.00.

DOI: https://doi.org/10.1145/3313831.3376196 groups (e.g. those with physical impairments), but a lack of tools means they can be excluded from completing these commonplace activities that many take for granted.

Many interface elements within common creative design applications (e.g. tool icons, alignment guides, etc.) have a visual angle spanning less than one degree. These tools can be efficiently selected with traditional input devices (i.e. a mouse), although research has shown that targets under this size are problematic to select via gaze $[10,44]$. This can be compounded further in creative applications where pixel level manipulations are essential in producing professional quality work. Interface zooming [1, 15, 62] and novel cursor control techniques [54] have been investigated to support small target selection. However, whilst these techniques can make existing interfaces more accessible, they involve "bolting-on" new features to existing interface paradigms optimized for traditional input devices.

This is unlikely to provide an optimal interaction experience for those who require the use of gaze tracking. It is therefore essential to investigate new interaction approaches for creative work that are tailored for those using gaze input. Initial work has started to explore how eye gaze can be used for facilitating basic artwork and drawing [13, 19, 23, 30, 43], although these applications present early investigations using simple features (e.g. allowing children to draw free-form colored lines). We therefore have little understanding about the optimal approaches for enabling people using gaze tracking technologies to undertake common graphical design activities (e.g. designing user interfaces, producing visual newsletters, digital illustrations, and website design).

We present a new application ("Sakura") that is tailored for the input combination of gaze pointing and a mechanical switch (which we define as "multimodal gaze" interaction). The prototype integrates key creative tools that have been adapted to make them more accessible for control via gaze and switch input. To demonstrate our design approach we provide an overview of the application and highlight the rationale behind the design of three specific features that have received little or no attention to date (in the context of visual creative design via multimodal gaze). These include object selection from a digital canvas (using a novel grid approach), the use of alignment guides to support object positioning, and approaches for object manipulation (in terms of size, location, color, etc.). 


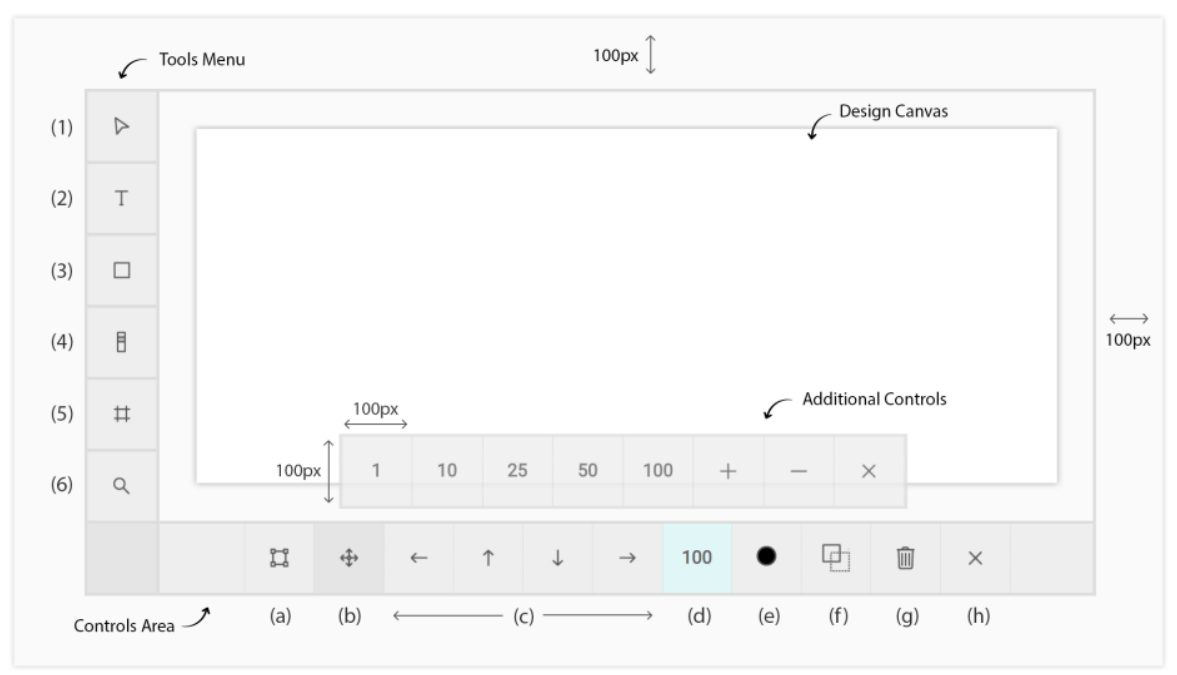

Figure 1: Screenshot of the Sakura interface. The "Tools Menu" contains six tools (from top-bottom) (1) Object Selection, (2) Text, (3) Shapes/Images, (4) Document Navigation, (5) Alignment Guides, and (6) Canvas Preview. The "Controls Area" contains object manipulation controls which include (from left-right) (a) Resize, (b) Move, (c) Directional Arrows, (d) Object Transformation Speed, (e) Color Selection, (f) Layers, (g) Delete Object, and (h) Close (which clears the "Controls Area").

We present results from an evaluation with 24 non-disabled participants - the application was rated highly in terms of usability and received positive feedback. A follow-up evaluation was conducted with six physically impaired participants where the application was again perceived positively and enabled them to work towards creating a new website design. Our work therefore presents three novel contributions - (1) a new application integrating multiple design features adapted for multimodal gaze interaction, (2) an evaluation highlighting new insights into the use of gaze interaction for visual design work, and (3) validation of our approach demonstrating that people with physical impairments can effectively manipulate graphical content.

\section{RELATED WORK}

\section{Gaze Applications for Creative Work}

The use of eye gaze tracking to support more creative tasks has received little attention to date. The earliest example is the work by Gips and Olivieri [13] where their "Eye Painting" application allowed children to draw colored lines on a screen wherever they looked. Whilst this approach demonstrated the ability to use our eyes for simple drawing, this type of free-from approach can be problematic for users who would like more control over their creative work. In particular, distinguishing between when the user would like to draw and when they are simply looking around the interface is a key issue (i.e. the Midas Touch issue [25]).

Hornof and Cavendar [22] explored a potential solution to this issue through their EyeDraw application that enabled users to signify intent to start drawing through a dwell-time selection. The final application included features such as adding basic shapes (rectangles, lines, ellipses, polygons) and color stamps to a canvas. However, whilst the application allowed children to draw basic images, it lacked many of the key features typically found in commercial design applications. The EyeArt application [42] also utilized a similar approach to EyeDraw where both a "watching" and "working" mode was used. Additional features included a wider set of shapes and the ability to enter text, although there are few details about how each of these features work and no usability evaluation is presented.

van der Kamp and Sundstedt [29] looked at the combination of eye gaze and speech input for producing graphical work. Their application made use of speech commands to start and stop the drawing process (as well as for selecting different tools), whereas gaze was used for controlling the position of the cursor. This application also allowed users to alter the color of shapes, although this functionality was limited as users could only select from a range of preset colors. Heikkilä [19] introduced the EyeSketch application that enables users to nudge, move, or resize basic shapes (through the support of a basic grid) via gaze gestures or dwell selections on control buttons. The application includes tools for adding basic shapes, deleting objects, and undoing previous actions. EyeSketch was therefore the first application that allowed users to select and manipulate existing objects on a canvas (via gaze), although objects were placed on a fixed grid thus constraining the possible location of graphical elements.

In addition to this research, there are also a range of gaze control applications and tools available such as Gaze Point [66], Windows Eye Control [43], and OptiKey [47] that enable mainstream software to be operated via controls that emulate mouse features (e.g. left, right, double clicks) and zooming techniques. However, whilst these tools can help to make certain applications more accessible, they do not provide tailored support for controlling specialist visual 


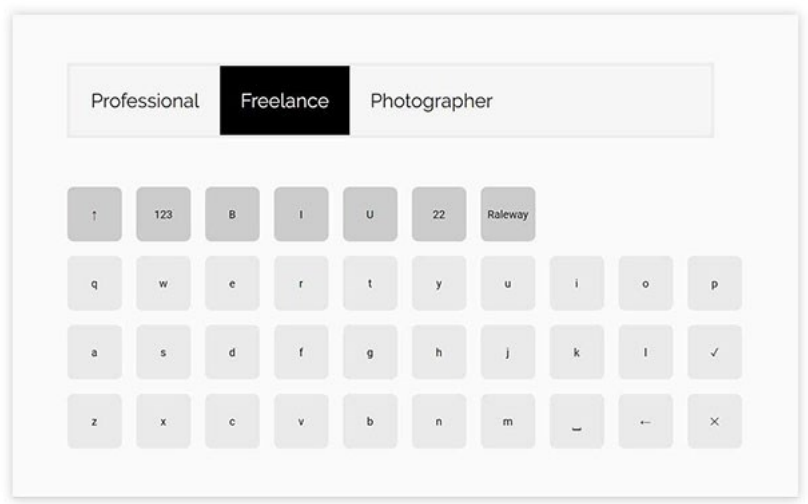

Figure 2: The onscreen keyboard and color selection tool.

creative design software (e.g. Adobe XD, Marvel, etc.) which require the frequent selection and manipulation of small graphical elements with pixel-level precision.

\section{Gaps in Knowledge}

Whilst the existing literature can help to inform the development of a multimodal gaze controlled visual design application, there still remain several areas where little or no work has been conducted - for instance:

1. Accurate Canvas Object Selection: The accurate selection of design artefacts (which can vary in shape, size, and orientation) located on a large design canvas.

2. Object Manipulation: The ability to manipulate graphical objects (e.g. text, images, and shapes) in a way that supports the development of more professional outputs (with pixel level precision).

3. Alignment Guides: The use of horizontal and vertical guides (commonly used in industry applications) to enable object snapping and alignment, thus helping to create higher quality creative work.

4. Color Selection: The selection of colors and use of palettes to facilitate the reuse of common colors in a design (as opposed to simpler approaches where users can select from a range of preset colors).

5. Creative Typography: Whilst the literature includes a significant focus on eye typing, no work has explored how to produce more artistic text using different fonts, text formatting, and font sizes.

Further research is therefore required to explore how these common features in creative design applications can be made more accessible for multimodal gaze interaction.

\section{APPLICATION DESIGN}

To address the gaps highlighted we developed a new visual creative design application ("Sakura") optimized for multimodal gaze interaction. We initially present the overall application interface and rationale for our design choices. We then provide three examples demonstrating how we adapted common design features to make them more accessible.

\section{Technical Details}

Sakura is a web-based prototype developed using HTML, CSS, and JavaScript. The application was designed to run in full-screen kiosk mode on Windows 10 and requires an eye tracker for pointing at interface elements, as well as the use of a mechanical switch for performing selections. Sakura is platform independent and will run in modern browsers with any eye tracker that allows for emulation of mouse movements. It can also work with other assistive technologies that provide users with control of the mouse cursor (e.g. head tracking, speech input, etc.).

\section{Overall Interface Design}

The main interface was informed through the design of existing applications focused around graphic design (Photoshop, InDesign, Inkscape), digital illustration (Illustrator, Corel Painter), and interface prototyping (Adobe XD, Sketch, Marvel, Axure). These applications often share common groupings within the interface such as a high-level menu area, a tools section, controls for tools, and panels for additional options (e.g. layers, color, alignment options, etc.). For example, Adobe XD (Figure 4) has a tools area on the left of the interface (for shapes, text, object selection, etc.), a main canvas area in the middle, and tool options on the right-side (e.g. for changing object colors, altering font sizes, etc.).

The Sakura interface is similarly divided into three key areas (Figure 1) - the "Tools Menu" provides tools for object selection (Figure 1 (1)), producing text (Figure 1 (2)), shape and image creation (Figure 1 (3)), scrolling the canvas (Figure 1 (4)), alignment guides (Figure 1 (5)), and a design preview (Figure 1 (6)). The main "Design Canvas" is located in the center, along with a "Tool Controls" area towards the bottom where controls are dynamically updated depending on the tool selected. An "Additional Controls" area includes extra options for some controls (e.g. object transformation sizes, layering options, etc.). Early usability testing found that users had issues selecting buttons located 


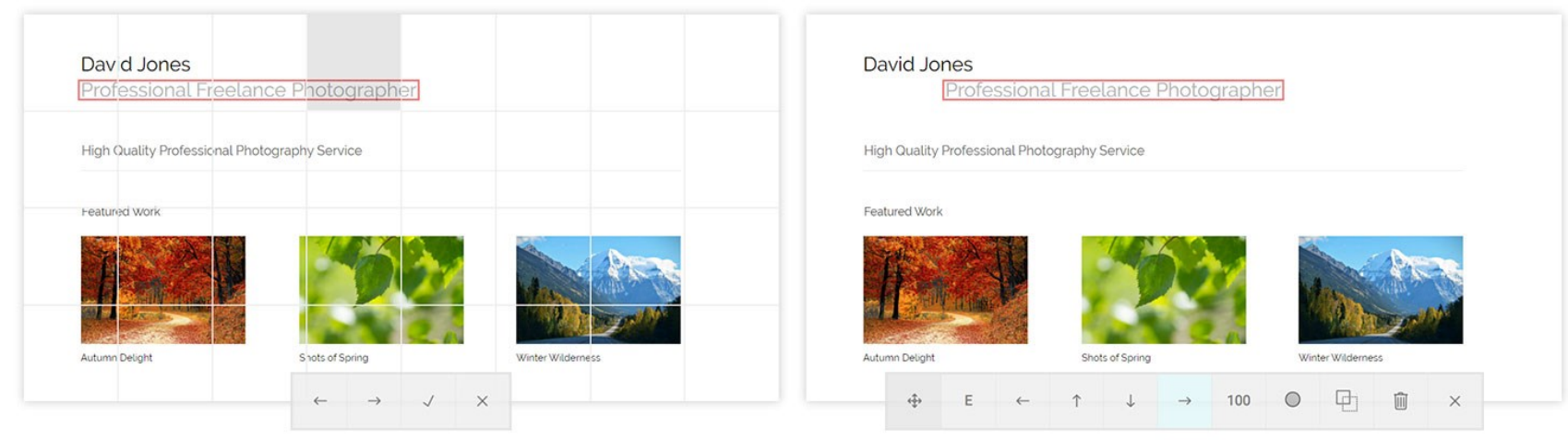

Figure 3: Screenshot of the object selection mode. In this example, the text "Professional Freelance Photographer" has been selected via the cell that is highlighted in gray. The user can then select the "tick" button to confirm their selection and then manipulate the object via the standard controls (in this case, the text has been moved 100px to the right).

close to the screen borders, so 100px padding was placed around the interface. All menu buttons were designed to be $100 \mathrm{x} 100 \mathrm{px}$ in size to enable more comfortable selection.

We took the design decision that objects placed on the main design canvas should not be interacted with directly via gaze. This was due to the inaccuracy of sensors which make performing pixel level selection and manipulation challenging. Instead, all object manipulations are performed through external options provided in the "Controls Area" to provide full control of canvas objects. The main canvas can also be scrolled using the document navigation tool (Figure 1 (4)) which provides up and down button controls, along with speed options (slow, medium, and fast). The canvas preview button (Figure 1 (6)) hides the main tools and extends the canvas vertically so users can preview more of their design at once.

The most common keyboard design used in eye typing studies is the standard QWERTY layout [32, 35, 36, 38, 39, 57]. This keyboard design was therefore incorporated into the application and can be displayed by choosing the "Text" tool (Figure 1 (2)). Additional buttons are placed above the main characters including a shift key, text formatting options, font size, and the font type. In terms of color selection, we opted for an adaptation of a standard color picker $[26,30,60]$ that presents a $2 \mathrm{D}$ color visualization combined with a slider (Figure 2). The interaction approach is consistent with the rest of the application in that colors can only be selected through controlling a circular cursor around the color space via external controls. Each time a color is selected it is automatically added to a palette to facilitate efficient reuse of colors.

To address the Midas Touch issue [25], numerous studies have explored and utilized dwell-based selections [12, 20, $36,39,48,57,58]$, although a downside of this method is the time required to perform a selection. Alternative selection approaches have also been explored (e.g. gaze gestures $[9,32,55,71]$, continuous zooming methods [16, 69], blinking [2], smiling [67], tooth clicking [72], pen tool and touch input [50-52]), but no work to date has explored the use of mechanical switches (commonly used by people with physical impairments) in combination with gaze. Sakura therefore supports the use of an external switch to trigger selections, thus providing an opportunity to explore this multimodal approach in the context of graphical design.

\section{Grid-Based Object Selection}

The ability to accurately select objects on a digital canvas is a fundamental requirement for creative work. Studies have explored different zooming methods that enable users to magnify an element of the interface to support selection of a small target $[1,4,6,15,17,31,45,62]$. Other approaches include the use of large controllable cursors to focus on areas of interest [54], as well as the use of gaze gestures to trigger selections [59]. However, no research to date has explored the use of these or alternative approaches for object selection in more creative pursuits where accurate selection can be confounded through the visual complexity of the interface (e.g. objects being a variety of shapes and sizes, distracting backgrounds, overlapping objects, etc.). One promising approach to aid the selection of targets is the use of grids that are overlaid on a digital canvas. Studies have investigated their use to support people with visual $[11,25,26,40,56]$ and physical impairments in using digital systems [44, 73], although no work has explored their potential for target selection in more "real-world" scenarios (i.e. from a digital canvas) using gaze pointing.

Sakura utilizes an $8 \times 4$ grid placed over the viewable canvas upon choosing of the "Object Selection" tool (Figure 1, (1)). This grid configuration results in cells that are over $100 \times 100$ px thus enabling more comfortable activation via gaze. To select an item on the canvas, users choose a cell which contains their desired object - the first object in that cell is highlighted with a red border around it. Users can then use left and right arrow controls to navigate between the different options within that cell. Once the desired object has been highlighted, users can confirm their choice (via a "tick" button) - the grid is then hidden and the standard object manipulation options are again displayed in 

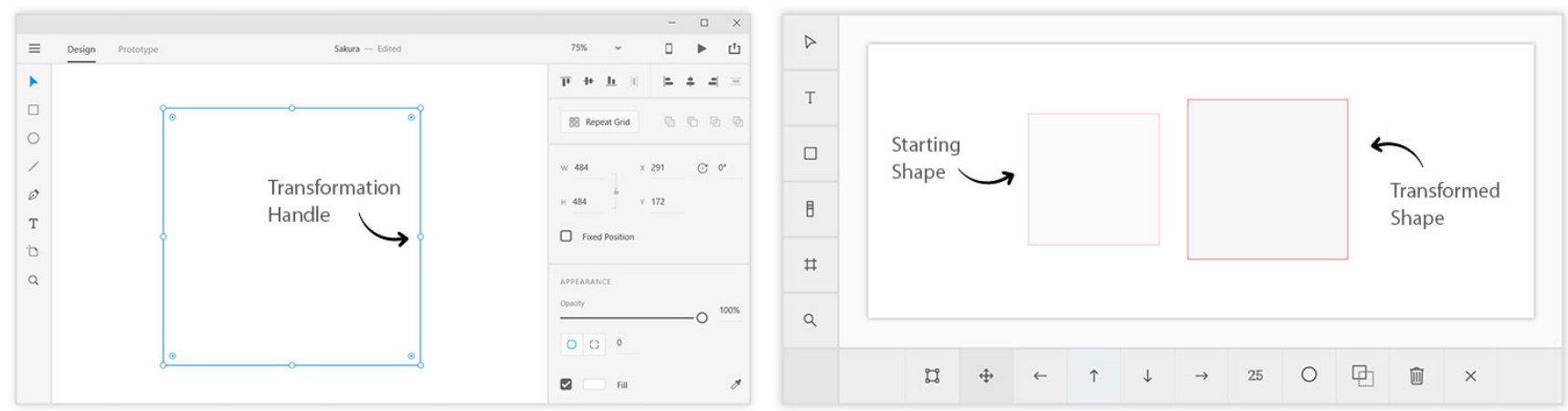

Figure 4: (Left) Screenshot of Adobe XD highlighting small transformation handles located directly on shapes; (Right) Screenshot of Sakura demonstrating a starting shape that has been transformed (i.e. resized and moved) using the controls available at the bottom of the interface (as opposed to object handles).

the "Controls Area" (Figure 3). This method provides potential benefits as it removes issues around target size influencing selection accuracy. Objects of any shape, size, orientation, or location can therefore be selected using a simple and consistent method that involves no distortion or magnification of the interface. This approach also represents a new gaze supported selection technique that has not been explored in the literature to date.

\section{Object Manipulation}

The size, location, and orientation of objects (i.e. text, images, shapes, etc.) in professional design applications are commonly manipulated through the use of small handles placed around the boundaries of an object (Figure 4). This approach can enable efficient manipulation of objects via mouse control, although it presents issues for those using gaze as their primary pointing method. Moreover, attempting to drag small handles to specific locations on a canvas is problematic due to the accuracy limitations of eye tracking [25, 44]. Existing research investigating the use of gaze interaction to support graphical work has yet to explore in detail how to address these issues. Heikkilä [19] is the only study to date that has examined the manipulation of the size and location of graphical objects. A gaze gesture approach was utilized in this work, although objects were placed on a fixed grid which constrained the possible location of graphical elements on a canvas.

Sakura utilizes controls external to an object to support manipulation of size and location (Figure 1a-1d). Users can move objects via the arrow keys when the "Move" control is enabled (Figure 1 (b)) - they can also access the "Resize" control (Figure 1 (a)) which supports resizing of objects using the same arrows. Transformation speed can be altered via the "Additional Controls" area (Figure 1) - this defaults to $100 \mathrm{px}$ with some other presets also provided (1px, 10px, $25 \mathrm{px}, 50 \mathrm{px})$. This provides a mix of smaller transformation options (for fine control) and larger speeds for more rapid manipulation. This method therefore supports full control over the location and size of objects without the difficulty in targeting pixel-level locations via direct gaze fixations.

\section{Alignment Guides}

The precise alignment of graphical objects is a crucial activity in design applications to facilitate the production of aesthetically pleasing designs. Grids are commonly used to snap graphical objects into alignment [41], along with horizontal and vertical alignment guides $[5,8,21]$. However, their use poses significant issues for eye gaze interaction as guides are typically very thin (Figure 5). Studies have explored the use of static fixed grids to provide some visual cues when drawing (via gaze) although these did not support object snapping [22, 42]. Heikkilä [18] used a fixed grid to support nudging, moving, and resizing of objects via gaze interaction - although the grid could not be customized. No work to date has therefore explored how to create and manipulate guides in design applications using gaze as the primary pointing approach.

Sakura provides a new method for manipulating alignment guides and snapping objects to them. Guides can be added to the canvas via selecting the "Alignment Guide" tool (Figure 1 (5)) - this presents controls for adding a horizontal or vertical guide (Figure 5). If, for example, the user adds a vertical guide, it is initially placed towards the left of the canvas. Users can then reposition the guide via left and right controls provided, along with speed options to support pixel level manipulation. To snap an object to a guide, users must (1) select the guide they wish to snap to, (2) choose the object they wish to place alongside the chosen guide, and (3) select the "Snap" option in the tool controls area. Figure 5 shows the process for snapping an image to a vertical guide - the same method also applies to horizontal guides. This approach therefore enables users to utilize thin alignment guides typically used in existing design applications, but manipulate them via external controls that are more accessible for multimodal gaze input.

\section{EVALUATION}

A user evaluation was conducted with non-disabled participants to investigate whether Sakura enabled users to complete common visual design activities. A key requirement for using the application is that all users are able to use their gaze to control the interface and operate a 


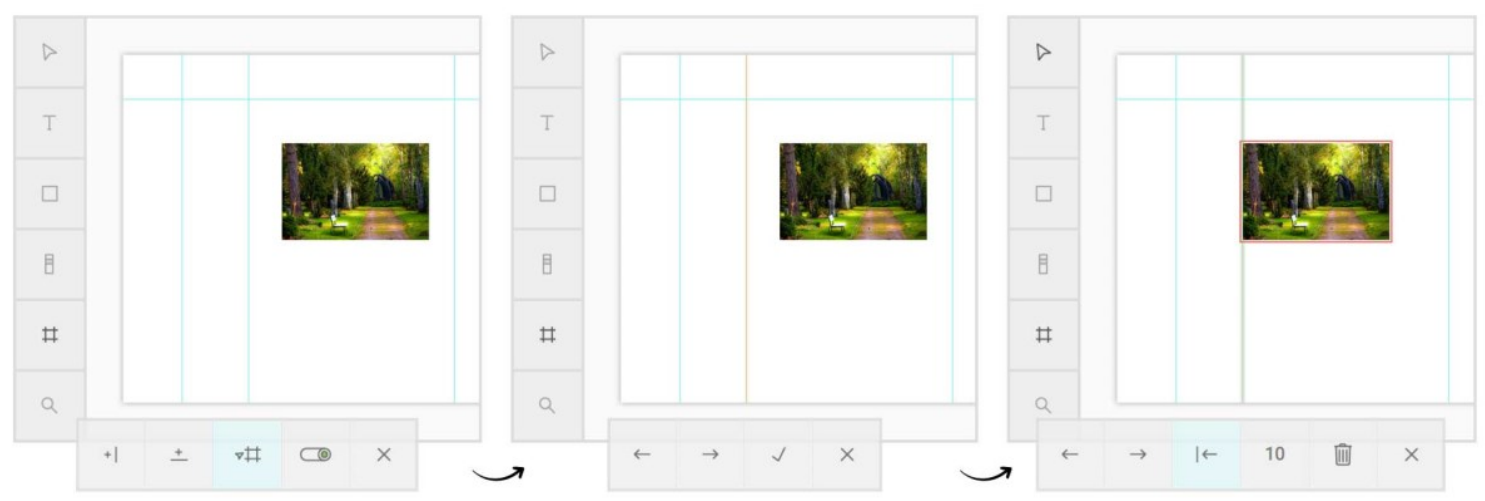

Figure 5: The process for snapping objects to guides - the first screenshot shows an image with some vertical and horizontal guides on the canvas. The middle screenshot provides an example of how a user has utilized the arrow keys to navigate to the second vertical guide (indicated by the orange glow around it). In the final screenshot the user has selected the image (via the object selection tool) and then used the "Snap" control (highlighted in blue) to snap the object to the guide.

switch for performing selections. It was therefore felt that a first study with non-disabled participants would provide a crucial and relevant insight into the use of this multimodal approach for graphical design work (prior to conducting evaluations with people who have physical impairments).

\section{Participants}

24 participants (10 female, 14 male) from a population of university students and staff members were recruited with a mean age of 23.6 years $(\mathrm{SD}=3.96) .8$ participants wore corrective lenses (all glasses). Participants completed a standardized consent form before the test and were not compensated. They were also asked to self-assess their level of experience with consumer design software, as well as alternative input methods for interfacing with computers:

- Experience with consumer interface design software (i.e. Adobe XD): 10 participants reported being novices while 12 reported to have an average knowledge and 2 selflabelled themselves as experts.

- Experience with image manipulation software (i.e. Photoshop, Gimp): 6 participants reported being novices while 16 stated they had an average knowledge and 2 self-reported themselves as experts.

- Experience with alternative input methods for interfacing with computers (i.e. tracking sensors, gestural interfaces): 15 participants categorized themselves as novices while 8 reported to have an average knowledge and the remaining 1 reported to be an expert.

No participants had any previous substantial experience with eye-tracking as an alternative input method.

\section{Apparatus}

Sakura was installed on a Windows 10 PC (Intel $\AA$ Core(TM) i7-6600U CPU and 16GB RAM) using a 22" monitor set to a resolution of 1920x1080px (with DPI scaling set to $125 \%$ ). The Eye Tribe eye tracker [65] was used to track eye movements which provides an average accuracy of 0.5 to $1^{\circ}$ of visual angle and an operating range between $45-75 \mathrm{~cm}$. The standard mouse cursor controls and stabilization features provided through the device's software were enabled to map the mouse cursor to participants' eye movements. The Eye Tribe tracker was attached to a small tripod placed in front of the monitor at a distance of approximately $60 \mathrm{~cm}$ from participants' eyes. A single $65 \mathrm{~mm}$ Jelly Bean switch was used to trigger selections and placed in front of the PC.

\section{Procedure}

Pre-Tasks: Participants were initially provided with an information sheet and were asked to provide informed consent. A survey was also administered to collect demographic information as well as details related to experience with existing consumer graphical editing software, eye tracking technologies, and other sensor based interactions. Participants were taken through a scripted walkthrough session which was controlled via the mouse by the researcher. A standard nine point calibration process was then completed using the Eye Tribe sensor. Following successful calibration, participants were able to practice with the application for between 5-10 minutes to ensure that they could comfortably control the interface.

Main Tasks: The research team developed a sample design using the application (Figure 6) which was then manipulated or "broken" in different ways to present users with tasks to fix or alter the design. There were four main tasks that participants were asked to complete which were comprised of multiple sub-tasks:

- Task 1: Add the text "Professional Freelance Photographer", change the font to "Raleway", font size to $24 \mathrm{px}$, and the color to gray. Position the text on the canvas under the main header (Figure 6 - area (a)).

- Task 2: Add a 1px line above the text "High Quality Professional Photography Service" and make the line blue (Figure 6 - area (b)); Select the 1px line below the text and make it the same color of blue (using the color 
palette tool); Add a gray background and place between these two lines and behind the text (using the rectangle and layer tools).

- Task 3: Select and delete the third image (on the right of the design); Add a new image (the one that contains mountains) and place it in the location of the previously deleted object; Make each of the three images 4px shorter in height (Figure 6 - area (c)).

- Task 4: Add a horizontal guide above the three images and snap each of the images to the guide; Add a new vertical guide and snap the blog post titles and dates to the new guide (Figure 6 - areas (d) and (e)).

Participants were initially shown the "starting" design on paper that would be seen upon starting the task. The researcher would then show a paper screenshot of the final design and outline the actions required to complete the task. Once participants had made the necessary updates, the researcher used a keyboard shortcut to move onto the next task where the process was repeated. The order of tasks was randomized to reduce the impact of any order effects.

In designing the evaluation our aim was not to create a highly controlled study, but instead to provide structured tasks that enabled participants to gain experience in using the features developed. This enabled us to explore if they were able to successfully complete common design activities and how they perceived the usability of the application. After participants had finished all tasks they were provided with access to an online survey to provide their views on each of the key features. Participants were finally asked to complete the System Usability Scale (SUS) survey [3] to assess the overall usability of Sakura.

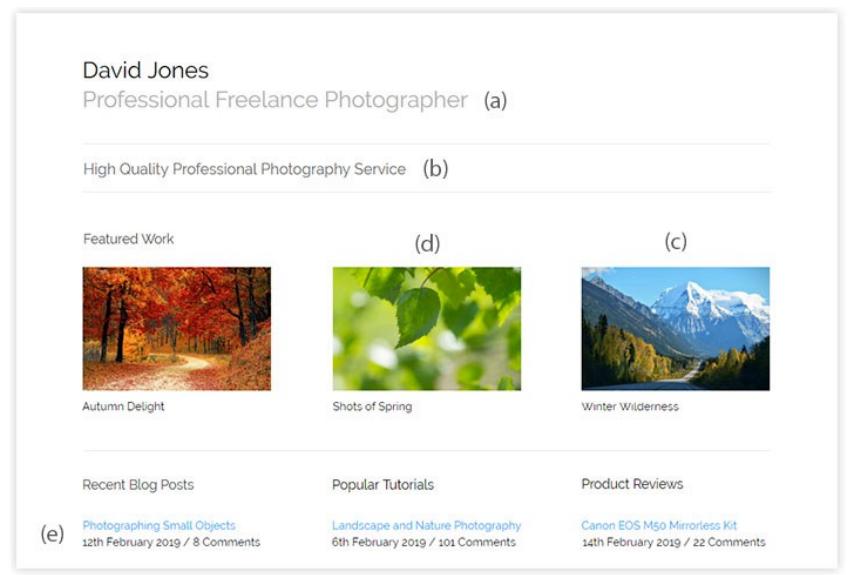

Figure 6: The website design used in the study - characters (a) - (e) highlight the key areas of focus for the evaluation tasks.

\section{Measures}

Task Completion Times: Task completion times were measured in milliseconds from when participants started each task (i.e. after the test coordinator had used the keyboard shortcut to initiate the task) until the required activities had been completed.
Perceived Usability: At the end of the study, participants were asked to complete an online survey with seven questions which focused on the main features of the application. SUS was also administered to obtain a sense of how participants perceived the usability of the application.

\section{Results}

\section{Task Completion Times}

The average completion time for the whole set of tasks was 22.9 minutes $(\mathrm{SD}=5.7 \mathrm{~min})$. Each task took under 8 minutes on average to complete, with Task1 requiring an average time of 4.16 minutes $(\mathrm{SD}=5.74 \mathrm{~min})$, Task2 7.05 minutes $(\mathrm{SD}=2.03 \mathrm{~min})$, Task3 4.08 minutes $(\mathrm{SD}=1.80$ $\mathrm{min})$ and Task4 5.48 minutes ( $\mathrm{SD}=2.62 \mathrm{~min})$. All participants were able to complete the presented tasks.

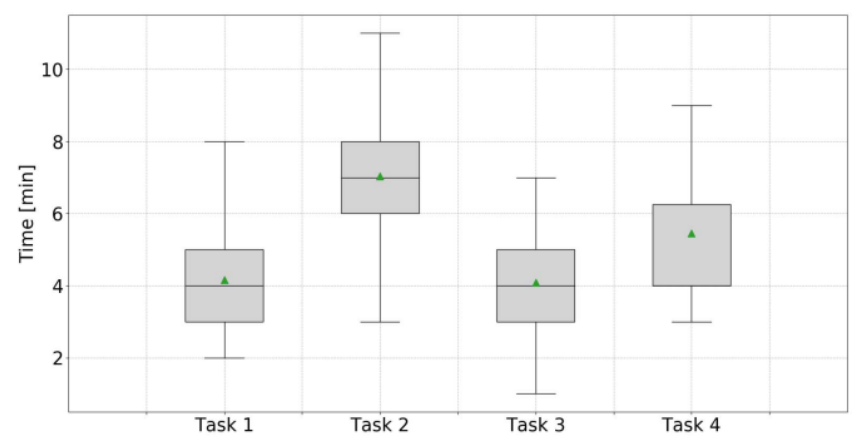

Figure 7: Completion times across each task.

\section{Usability (SUS)}

The overall design and implementation of the Sakura system obtained an average SUS score of 82.39 (SD = 11.12). This score can be labelled as good and usable according to Bangor et al. [3].

\section{Survey Analysis}

Object Selection: The majority of participants provided positive comments around selecting items via the grid tool (22 participants). These positive comments tended to emphasize that the approach was fast and efficient to use: "I liked the grid based approach as it allowed for much more controlled input. The ability to scroll through the objects within a small zone using a button approach also worked well." (Participant 16). Three participants also commented that the large size of the cell buttons help to support their desired selection. Thirteen participants highlighted some frustrations with the most common issue ( 7 participants) being the need to switch between gazing on the current button (in the tool controls area) and then shifting their gaze back to the canvas to check the updates that had been made: "... it does suffer from the instinct to look at what object you highlighted when you scroll through multiple objects within a small space." (Participant 17).

Object Manipulation: Twenty participants stated positive comments around the adding and manipulation of shapes on 
Table 1: Participant details and SUS scores (Technical Details Key - ID: Interface Design; GM: Graphical Manipulation; AM: Alternative Input Methods; ET: Eye tracking)

\begin{tabular}{|c|c|c|c|c|}
\hline$I D$ & Age & Physical Impairments & Technical Details & $S U S$ \\
\hline $\mathrm{P} 1$ & $70(\mathrm{M})$ & $\begin{array}{l}\text { MND (since 2014). Muscle weakness on left side; Non-verbal (since 2018); } \\
\text { good level of control on right side of body. Uses a powered chair. }\end{array}$ & $\begin{array}{l}\text { ID: Novice; GM: Average; } \\
\text { AM: Novice; ET: Novice. }\end{array}$ & 75 \\
\hline $\mathrm{P} 2$ & $63(\mathrm{M})$ & $\begin{array}{l}\text { MND (since 2011). Lack of balance; Difficulty with speaking. Uses Grid Pad } \\
\text { [14] with a tablet, although has little strength in hands. Uses a powered chair. }\end{array}$ & $\begin{array}{l}\text { ID: Novice; GM: Average; } \\
\text { AM: Average; ET: Novice. }\end{array}$ & 60 \\
\hline P3 & $72(\mathrm{M})$ & $\begin{array}{l}\text { MND (since 2019). Difficulty with walking and arm movements; Weak } \\
\text { dexterity and developing difficulty with speech. }\end{array}$ & $\begin{array}{l}\text { ID: Average; GM: Expert; } \\
\text { AM: Novice; ET: Novice. }\end{array}$ & 67.5 \\
\hline P4 & $62(\mathrm{M})$ & $\begin{array}{l}\text { MND (since } 2015 \text { ). Weakness in arms and legs; Difficulty with speech; Uses a } \\
\text { powered chair for mobility. }\end{array}$ & $\begin{array}{l}\text { ID: Average; GM: Average; } \\
\text { AM: Novice; ET: Novice. }\end{array}$ & 80 \\
\hline P5 & $74(\mathrm{~F})$ & $\begin{array}{l}\text { MND (since 2017). Non-verbal, uses tablet for communication (text to speech); } \\
\text { Cannot fully control all fingers on right-hand; Difficulty holding neck up. }\end{array}$ & $\begin{array}{l}\text { ID: Novice; GM: Expert; } \\
\text { AM: Novice; ET: Novice. }\end{array}$ & 77.5 \\
\hline P6 & $53(\mathrm{M})$ & $\begin{array}{l}\text { MS (since 2014). Relapse/Remitting. Experiences fatigue and poor mobility. } \\
\text { Fine control is deteriorating - drawing straight lines is becoming more difficult. }\end{array}$ & $\begin{array}{l}\text { ID: Average; GM: Expert; } \\
\text { AM: Average; ET: Novice. }\end{array}$ & 82.5 \\
\hline
\end{tabular}

the design canvas. Comments focused around the controls being simple, easy, and intuitive to use: "The speed feature was useful as it allowed me to position the images to the locations I wanted. The moving feature was quite intuitive and simple to use." (Participant 12). A common problem highlighted by nine participants were issues between knowing when the resize larger or smaller tool was selected (thus leading to some unintentional object transformations): "One of the most common mistakes I made while resizing was forgetting to change the mode from increase size to decrease size. As I am generally looking at the buttons when re-sizing images I do not notice that I am editing wrong until I have made several steps." (Participant 1).

Alignment Guides: 19 participants provided positive feedback on the alignment guide feature in that it was fast to snap objects, easy to use, and a helpful tool for aligning objects: "I think this is probably the most handy and useful part of the entire application as it makes the application much easier to use especially for aligning objects and text" (Participant 5). Three participants commented that snapping objects was time consuming and involved numerous button presses: "This would be a very useful feature, but I didn't like how I had to exit the interface each time to snap things to the grid (this was not actually necessary), it seemed to take too many button presses when there were several objects which needed to be aligned." (Participant 3). Three participants also highlighted that having to switch their gaze between the tool controls area and main canvas when selecting a guide was an issue (Participants 5, 10, 14).

General Comments: Further comments re-emphasized participants overall positive perceptions of the prototype and ability to complete the tasks. For instance, Participant 14 commented that they "... enjoyed using this system and found the tasks to be easier than initially expected. It felt like the system had all the fundamental functionality required to perform most standard design tasks and it felt quite intuitive to use and easy to learn.". Similarly, Participant 25 highlighted that "... the system is very well designed and has really considered how best to function and how the user would navigate through the options".

\section{FOLLOW-UP STUDY}

A follow-up study was conducted with six participants who have physical impairments to explore their experiences in using the application.

Participants: 6 participants (5 male and 1 female) were recruited via an online advertisement made in partnership with the Motor Neurone Disease Association [46] and through existing links with the research team. Participants had a mean age of 65.7 years $(\mathrm{SD}=7.9)$ with five being diagnosed with Motor Neurone Disease (MND) and one with Multiple Sclerosis (MS). Participants were asked to self-assess their level of experience with consumer design software and alternative input methods (Table 1). Four participants were naïve to eye tracking technology except for P5 who had previously tested the technology (in a short five minute session) and P6 who had been involved with a previous (unrelated) research study. Both participants still self-identified as being novices with the technology. Five participants wore corrective lenses (all glasses).

Apparatus: Sakura was installed on a 15.6" Lenovo ThinkPad laptop (Intel® Core(TM) i7-6600U CPU and 16GB RAM) running Windows 10 . The screen resolution was set to $1920 \times 1080$ px with DPI scaling set to $125 \%$. The eye tracker was attached to the bottom of the screen using a magnetic connector. A $65 \mathrm{~mm}$ Jelly Bean switch was used to trigger selections and placed in front of the laptop (Figure 8). The equipment was set up on a table and tailored to participants' needs (e.g. P1 only had movement on his right side, so the switch was placed to the right of the laptop). 
Procedure: Institutional Review Board (IRB) approval was initially obtained for the study. Evaluations were conducted in the participant's home or work environment (P1, P3, P5, P6), our lab (P2), or at a social meetup for people with MND (P4). Caregivers were also present during the study and encouraged to share their views. Participants were given a study information sheet and asked to provide informed consent. An initial survey was then administered to collect demographic information, details of impairments, and information around their technical background. Participants were given a guided walkthrough of the system and the eye tracker calibration process was then completed. They then worked towards creating the website design in Figure 6 (provided on paper for reference) from a blank canvas, although they were encouraged to adapt the design however they preferred.

The core aim of this task was to give participants a starting template to work towards and to encourage use of the main tools (as opposed to completing a full design). A screenshot of each participant's final design was taken once they had used the key features and at least added some text, alignment guides, and images. A semi-structured interview was then conducted to collect participants' views. Sessions lasted between 90-120 minutes and participants were informed that they could take breaks or withdraw from the study at any time. Participants were not compensated, although we offered to cover travel expenses or to visit them at another location if they were unable to travel.

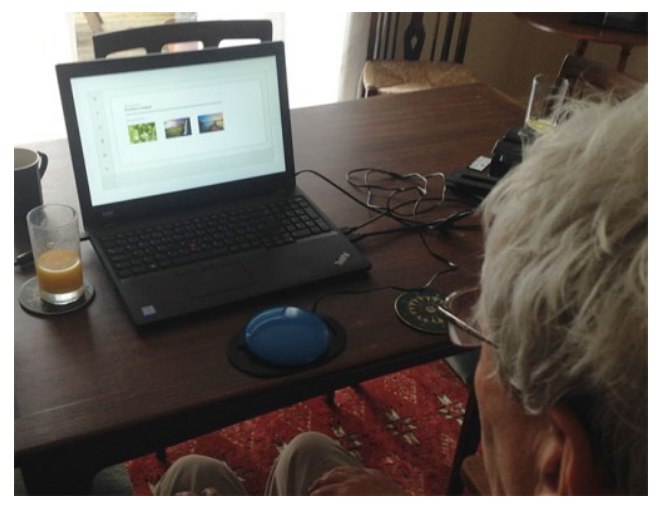

Figure 8. A participant using the application in their home.

Results

Usability (SUS): Sakura obtained an average SUS score of $73.75(\mathrm{SD}=8.55)$. This score can be labelled as good and usable according to Bangor et al. [3]. Four participants provided scores of 75 or over with two participants who identified as having expert experience in graphical design providing the highest scores (P5 - a professional photographer; P6 - a professional illustrator). The lowest scores were provided by P2 (60) and P3 (67.5) which can be labelled as "OK" in terms of usability [3].

Design Outputs: All participants were able to utilize the features within Sakura to create an initial website design.
P5 and P6 produced designs that contained more canvas elements (i.e. text, images, shapes, etc.) and made more extensive use of alignment guides. For instance, Figure 9 provides a screenshot of $\mathrm{P} 6$ 's design which took 50 minutes to create. This screenshot demonstrates how P6 utilized alignment guides, different typography and colors, lines, images, and use of the application's controls to manipulate the location of these objects. P6 commented that the application was intuitive, simple, and straightforward to use. Other participants (P1, P2, P3, and P4) were also able to use the tools available although their final design typically incorporated fewer design elements (e.g. a couple of alignment guides, three images, and some text - i.e. the name/subtitle). The times to create these designs ranged from 29 minutes (P4) - 59 minutes (P3).

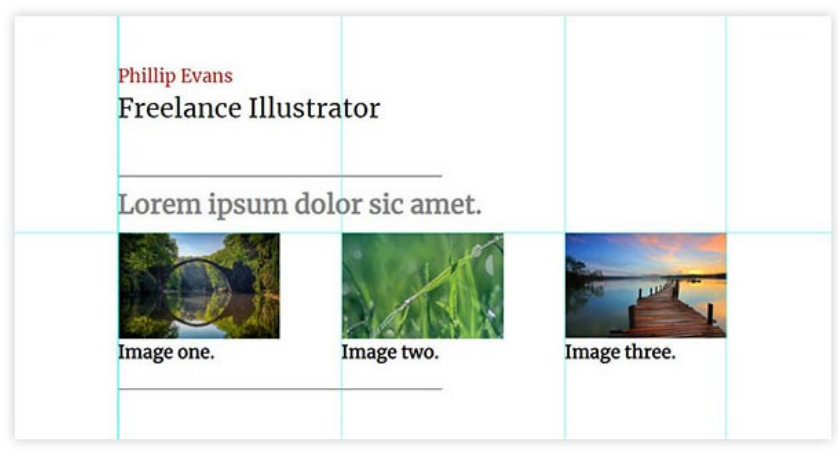

Figure 9: P6's final design screenshot.

Button Selection Issues: 4 participants (P1, P2, P3, P4) experienced issues in selecting tool controls and keyboard buttons. This was a particular issue when typing text as participants had a tendency to look away at the next letter before releasing the switch. The dexterity of these participants' hands also contributed towards this issue as it was challenging to perform quick taps of the switch. P5 did not experience significant issues with controlling the interface, although she commented that the switch was a little "heavy" and required effort to use (which resulted in her right hand becoming tired).

Location of Keyboard Buttons: The positioning of some keyboard buttons created an issue for P1, P2, and P4. For instance - when attempting to select the backspace key, P2 would often accidently select the space key instead. This added a significant interaction cost as the space would then need to be deleted, in addition to the original correction. P2 also selected the "Close Keyboard" key on occasions (which is in close proximity to the "Add Text" button) when typing which led to the text being deleted and a return to the main canvas. However, it is important to note these issues did not apply for all participants (e.g. P5 and P6).

Disconnect Between Controls and Canvas Objects: All 6 participants re-iterated an issue from the first study in that it is difficult to see manipulations being performed on an object when focusing their gaze on a tool control. This was also an issue when participants entered an incorrect letter 
during typing - they would often continue typing and later realize that a "mistake" had been made. This added a further interaction cost as more characters had to be corrected to achieve the desired result.

Starting Position of Objects: P5 and P6 commented that the default starting position of newly created objects towards the top-left of the canvas was not an ideal approach. This was particularly an issue when objects had to be moved larger distances. They suggested an alternative approach would be to first gaze where they would approximately like the object to be placed and then "nudging" it into a specific location (via the controls provided).

\section{DISCUSSION}

The paper presents an evaluation of Sakura, a multimodal gaze interaction system integrating multiple common visual creative design tools that have been adapted to enhance their accessibility for people with physical impairments. Qualitative feedback across both studies was very positive about the different features with SUS scores indicating that the system has a good level of usability [3]. Participants therefore found manipulating canvas objects via external controls (i.e. arrow buttons, speed options, etc.) to be a simple, usable, and intuitive interaction approach.

The gaze interaction literature has predominantly focused on addressing isolated content creation and user interface design challenges. Eye typing has extensively been addressed separately $[32,35,36,38,39,57]$, creative applications have predominantly addressed drawing features only $[11,19,32,17,21]$, and object selection via dwell-based and other approaches have been explored as a single challenge $[33,36,49,61]$. However, there is a current lack of studies and system development work addressing more than one specific creative input feature and integrating them in a usable interface. This paper has presented such a system, enabling prospective creative professionals to design, layout, and edit graphical work. This work therefore addresses key challenges in the use of multimodal gaze as an interaction approach for the creation of visual design work, while presenting a fully functional user interface for creative activities that provides a usable solution for people with physical impairments.

\section{Limitations}

One limitation of the work is that participants across both evaluations only completed the defined tasks once - it will be important in future work to conduct a longitudinal study and set additional design challenges for participants. However, given the positive responses, we feel that increased familiarity and exposure to the system will enable users to more rapidly complete visual design work. There is little research addressing the use of grids for selecting and snapping objects via gaze [42], with no previous work enabling the adding and editing of alignment guides. Whilst many users were positive about this functionality, other methods need to be explored to potentially simplify this process (e.g. approaches that enable participants to snap objects directly from the object manipulation controls area). Some participants reported that when they are fixating on a tool control (e.g. the arrows), they cannot see the transformations being performed on the main canvas. It may be that providing controls "in situ" (i.e. close to the object selected) may be a better approach - although these buttons would likely obscure other artefacts on the canvas. It will be important to explore alternative approaches as this was a common theme emerging from the evaluations.

\section{Future work}

The following new features were identified as being of interest for participants across both studies: (1) the ability to multi-select objects on the canvas (via gaze input) and perform a series of transformations simultaneously, (2) the ability to snap the size of objects to guides (as opposed to just their location) could help make it easier and more efficient to produce objects that match in size, (3) the ability to copy and paste existing objects via gaze interaction is also a common design activity, although has received no attention from researchers, (4) more options for editing typographical errors as opposed to a simple backspace key that is typically used in eye typing studies $[20,23,24,34]$, and (5) the use of a pen tool and the creation of other custom shapes via gaze input.

The use of magnification and zooming within a digital canvas will also potentially be beneficial when utilizing certain new features (e.g. a gaze supported pen tool). Further work investigating how this functionality can effectively be incorporated into creative applications (utilizing a multimodal gaze approach) is therefore another key research area. In terms of multimodal approaches, there is the opportunity to combine gaze interaction with other input mechanisms to explore how this influences use of the application. For example, speech input could be used to trigger different features (e.g. "Select") and gaze could then be used for object manipulation work [29]. This would potentially enable the current menus within the interface to be hidden thus freeing up more space for the design canvas.

\section{CONCLUSION}

We introduced a new application "Sakura" that enables people with physical impairments to complete visual design work via a multimodal gaze interaction approach. The paper introduces new techniques for the selection of design artefacts located on design canvases, object manipulation, and the alignment of graphical objects using guides. Sakura is the first application to integrate each of these features into a single multimodal gaze controlled system and to explore the creation of web design work via this method of interaction (as opposed to basic artwork). User evaluations across two studies found that the application was rated positively in terms of usability and highlighted several interesting areas for future research that can help develop further activity in this important and underexplored field. 


\section{REFERENCES}

[1] Michael Ashmore, Andrew Duchowski, and Garth Shoemaker. 2005. Efficient Eye Pointing with a Fisheye Lens Michael. In Proceedings of Graphics Interface, 203-210.

[2] Behrooz Ashtiani and I. Scott MacKenzie. 2010. BlinkWrite2: an improved text entry method using eye blinks. In Proceedings of the 2010 Symposium on EyeTracking Research \& Applications, 339-345.

[3] Aaron Bangor, Philip Kortum, and James Miller. 2009. Determining what individual SUS scores mean: Adding an adjective rating scale. Journal of Usability Studies 4, 3: 114-123.

[4] Richard Bates and Howell Istance 2002. Zooming interfaces!: enhancing the performance of eye controlled pointing devices. In Proceedings of the fifth international ACM conference on Assistive technologies, 119-126.

[5] Patrick Baudisch, Edward Cutrell, Ken Hinckley, and Adam Eversole. "nap-and-go: helping users align objects without the modality of traditional snapping. In Proceedings of the SIGCHI conference on Human factors in computing systems, 301-310.

[6] T.R. Beelders and P.J. Blignaut. 2011. The Usability of Speech and Eye Gaze as a Multimodal Interface for a Word Processor. In Speech Technologies, Ivo Ipsic, IntechOpen.

[7] Ralf Biedert, Georg Buscher, and Andreas Dengel. 2010. The eyebook-using eye tracking to enhance the reading experience. Informatik-Spektrum 33, 3: 272281.

[8] Eric A. Bier and Maureen C. Stone. 1986. Snapdragging. In ACM SIGGRAPH Computer Graphics, 233-240.

[9] Tuhin Chakraborty, Sayan Sarcar, and Debasis Samanta. 2014. Design and evaluation of a dwell-free eye typing technique. In Proceedings of the extended abstracts of the 32nd annual ACM conference on Human factors in computing systems, 1573-1578.

[10] Chris Creed. 2016. Assistive tools for disability arts: collaborative experiences in working with disabled artists and stakeholders. Journal of Assistive Technologies 10, 2: 121-129.

[11]Liwei Dai, Rich Goldman, Andrew Sears, and Jeremy Lozier. 2005. Speech-based cursor control using grids: modelling performance and comparisons with other solutions. Behaviour \& Information Technology 24, 3: 219-230.

[12] Antonio Diaz-Tula and Carlos Morimoto. 2016. AugKey: Increasing Foveal Throughput in Eye Typing with Augmented Keys. In Proceedings of the 2016 CHI conference on human factors in computing systems, 3533-3544.
[13] James Gips and Peter Olivieri. 1996. EagleEyes: An eye control system for persons with disabilities. In Proceedings of the eleventh international conference on technology and persons with disabilities, 1-15.

[14] Smartbox. 2019. Grid Pad. Retrieved September 19, 2019 from https://thinksmartbox.com/product/grid-pad/

[15] Yasmin Halwani, Septimiu Salcudean, Victoria Lessoway, and Sidney Fels. 2017. Enhancing Zoom and Pan in Ultrasound Machines with a Multimodal Gaze-based Interface. In Proceedings of the 2017 CHI Conference Extended Abstracts on Human Factors in Computing Systems, 1648-1654.

[16] Dan Hansen, Henrik Skovsgaard, John Hansen, and Emilie Møllenbach. 2008. Noise tolerant selection by gaze-controlled pan and zoom in 3D. In Proceedings of the 2008 symposium on Eye tracking research \& applications, 205-212.

[17] John Hansen, Javier Agustin, and Henrik Skovsgaard. 2011. Gaze Interaction From Bed. In Proceedings of the 1st Conference on Novel Gaze-Controlled Applications, 11.

[18]Henna Heikkilä. 2013. EyeSketch: a drawing application for gaze control. In Proceedings of the 2013 Conference on Eye Tracking South Africa, 71-74.

[19] Henna Heikkilä. 2013. Tools for a Gaze-Controlled Drawing Application-Comparing Gaze Gestures against Dwell Buttons. In Proceedings of IFIP Conference on Human-Computer Interaction, 187-201.

[20] Jens Helmert, Sebastian Pannasch, and Boris Velichkovsky. 2008. Influences of dwell time and cursor control on the performance in gaze driven typing. Journal of Eye Movement 2, 4: 1-8.

[21] Seongkook Heo, Yong-Ki Lee, Jiho Yeom, and Geehyuk Lee. 2012. Design of a shape dependent snapping algorithm. In CHI'12 Extended Abstracts on Human Factors in Computing Systems, 2207-2212.

[22] Anthony Hornof and Anna Cavender. 2005. EyeDraw: enabling children with severe motor impairments to draw with their eyes. In Proceedings of the SIGCHI conference on Human factors in computing systems, 161-170.

[23] Anke Huckauf and Mario H. Urbina. 2008. Gazing with pEYEs: towards a universal input for various applications. In Proceedings of the 2008 symposium on Eye tracking research \& applications, 51-54.

[24]Kenji Itoh, Hirotaka Aoki, and John Paulin Hansen. 2006. A comparative usability study of two Japanese gaze typing systems. In Proceedings of the 2006 symposium on Eye tracking research \& applications, 59-66.

[25] Robert Jacob and Keith Karn. 2003. Eye tracking in human-computer interaction and usability research: Ready to deliver the promises. In The Mind's Eye, 573- 
605.

[26] Ghita Jalal, Nolwenn Maudet, and Wendy E. Mackay. 2015. Color portraits: From color picking to interacting with color. In Proceedings of the 33rd Annual ACM Conference on Human Factors in Computing Systems, 4207-4216.

[27] Hesham M. Kamel and James A. Landay. 2000. A study of blind drawing practice: creating graphical information without the visual channel. In Proceedings of the fourth international ACM conference on Assistive technologies, 34-41.

[28] Hesham M. Kamel and Halil I. Erhan. 2013. WebSight: the use of the grid-based interface to convey layout of web-pages in a non-visual environment. In International Conference on Universal Access in Human-Computer Interaction, 674-683.

[29] Jan van der Kamp and Veronica Sundstedt. 2011. Gaze and voice controlled drawing. In Proceedings of the 1st Conference on Novel Gaze-Controlled Applications, 18.

[30]EunJin Kim and Hyeon-Jeong Suk. 2017. Thoughts and Tools for Crafting Colors: Implications from Designers' Behavior. In Proceedings of the 2017 Conference on Designing Interactive Systems, 321-331.

[31] Manu Kumar, Terry Winograd, and Andreas Paepcke. 2007. Gaze-enhanced Scrolling Techniques. Gazeenhanced scrolling techniques. In Proceedings of CHI'07 Extended Abstracts on Human Factors in Computing Systems, 2531-2536.

[32] Andrew Kurauchi, Wenxin Feng, Ajjen Joshi, Carlos Morimoto, and Margrit Betke. 2016. EyeSwipe: Dwellfree Text Entry Using Gaze Paths. In Proceedings of the 2016 CHI Conference on Human Factors in Computing Systems, 1952-1956.

[33] Chris Lankford. 2000. Effective eye-gaze input into windows. In Proceedings of the 2000 symposium on Eye tracking research \& applications, 23-27.

[34] Shuo Samuel Liu, Andrew Rawicz, Teng Ma, Cheng Zhang, Kyle Lin, Siavash Rezaei, and Eion Wu. 2010. An eye-gaze tracking and human computer interface system for people with ALS and other locked-in diseases. CMBES 33, 1.

[35] I. Scott MacKenzie and Xuang Zhang. 2008. Eye typing using word and letter prediction and a fixation algorithm. In Proceedings of the 2008 symposium on Eye tracking research \& applications, 55-58.

[36]Päivi Majaranta, Ulla-Kaija Ahola, and Oleg Špakov. 2009. Fast gaze typing with an adjustable dwell time. In Proceedings of the 27th international conference on Human factors in computing systems, 357-360.

[37] Päivi Majaranta and Andreas Bulling. 2014. Eye tracking and eye-based human-computer interaction. Advances in physiological computing, 39-65.
[38] Päivi Majaranta, Poika Isokoski, Jussi Rantala, and Oleg Špakov. 2016. Haptic feedback in eye typing. Journal of Eye Movement Research 9, 1: 1-14.

[39] Päivi Majaranta, I. Scott MacKenzie, Anne Aula, and Kari-Jouko Räihä. 2006. Effects of feedback and dwell time on eye typing speed and accuracy. Universal Access in the Information Society 5, 2: 199-208.

[40]Päivi Majaranta and Kari-Jouko Räihä. 2002. Twenty years of eye typing: systems and design issues. In Proceedings of Eye Tracking Research and Applications, 15-22.

[41] Toshiyuki Masui. HyperSnapping. 2001. In Proceedings IEEE Symposia on Human-Centric Computing Languages and Environments, 188-194.

[42] André Meyer and Markus Dittmar. 2009. Conception and development of an accessible application for producing images by gaze interaction - EyeArt.

[43] Microsoft. 2019. Eye Control in Windows 10. Retrieved December 20, 2019 from https://support.microsoft.com/en-us/help/4512610.

[44] Darius Miniotas, Oleg Špakov, Ivan Tugoy, and I. Scott MacKenzie. 2006. Speech-augmented eye gaze interaction with small closely spaced targets. In Proceedings of the 2006 symposium on Eye tracking research \& applications, 67-72.

[45] Emilie Mollenbach, Thorarinn Stefansson, and John P. Hansen. 2008. All eyes on the monitor. In Proceedings of the 13th international conference on Intelligent user interfaces, 373-376.

[46] MNDA. 2019. Motor Neurone Disease Association. Retrieved September 19, 2019 from https://www.mndassociation.org/.

[47] OptiKey. 2019. Optikey Website. Retrieved December 20, 2019 from https://bit.ly/2QcLCoq.

[48] Prateek Panwar, Sayan Sarcar, Debasis Samanta. 2012. EyeBoard: A fast and accurate eye gaze-based text entry system. In Proceedings of 4th International Conference on Intelligent Human Computer Interaction (IHCI), 1-8.

[49] Abdul Moiz Penkar, Christof Lutteroth, and Gerald Weber. 2012. Designing for the eye: design parameters for dwell in gaze interaction. In Proceedings of the 24th Australian Computer-Human Interaction Conference, 479-488.

[50] Ken Pfeuffer, Jason Alexander, Ming Ki Chong, and Hans Gellersen. 2014. Gaze-touch: combining gaze with multi-touch for interaction on the same surface. In Proceedings of the 27th annual ACM symposium on User interface software and technology, 509-518.

[51] Ken Pfeuffer, Jason Alexander, Ming Ki Chong, Yanxia Zhang, and Hans Gellersen. 2015. Gazeshifting: Direct-indirect input with pen and touch 
modulated by gaze. In Proceedings of the 28th Annual ACM Symposium on User Interface Software \& Technology, 73-383.

[52] Ken Pfeuffer and Hans Gellersen. 2016. Gaze and touch interaction on tablets. In Proceedings of the 29th Annual Symposium on User Interface Software and Technology, 301-311.

[53] Marco Porta and Alessia Ravelli. 2009. WeyeB, an eye-controlled web browser for hands-free navigation. In 2nd Conference on Human System Interactions, 210215.

[54] Marco Porta, Alice Ravarelli, Giovanni Spagnoli. 2010. ceCursor, a contextual eye cursor for general pointing in windows environments. In Proceedings of the 2010 Symposium on Eye-Tracking Research \& Applications, 331-337.

[55] Marco Porta and Matteo Turina. 2008. Eye-S: a fullscreen input modality for pure eye-based communication. In Proceedings of the 2008 symposium on Eye tracking research \& applications, 27-34.

[56] Kari-Jouko Räihä and Selina Sharmin. 2014. Gazecontingent scrolling and reading patterns. In Proceedings of the 8th Nordic Conference on HumanComputer Interaction: Fun, Fast, Foundational, 65-68.

[57] Kari-Jouko Räihä and Saila Ovaska. 2012. An exploratory study of eye typing fundamentals: dwell time, text entry rate, errors, and workload. In Proceedings of the SIGCHI conference on human factors in computing systems, 3001-3010.

[58] Sayan Sarcar and Prateek Panwar. 2013. Eyeboard++. In Proceedings of the 11th Asia Pacific Conference on Computer Human Interaction, 354-363.

[59] Simon Schenk, Marc Dreiser, Gerhard Rigoll, and Michael Dorr. 2017. GazeEverywhere: Enabling Gazeonly User Interaction on an Unmodified Desktop PC in Everyday Scenarios. In Proceedings of the 2017 CHI Conference on Human Factors in Computing Systems, 3034-3044.

[60] Maria Shugrina, Wenjia Zhang, Fanny Chevalier, Sanja Fidler, and Karan Singh. 2019. Color Builder: A Direct Manipulation Interface for Versatile Color Theme Authoring. In Proceedings of the 2019 CHI Conference on Human Factors in Computing Systems, Paper No: 456.

[61] Linda Sibert and Robert Jacob. 2000. Evaluation of eye gaze interaction. In Proceedings of the SIGCHI conference on Human Factors in Computing Systems, 281-288.

[62] Henrik Skovsgaard, Julio Mateo, John Flach, and John Paulin Hansen. 2010. Small-target selection with gaze alone. In Proceedings of the 2010 Symposium on EyeTracking Research \& Applications, 145-148.

[63] Henrik Skovsgaard, Julio Mateo, and John Paulin
Hansen. 2011. Evaluating gaze-based interface tools to facilitate point-and-select tasks with small targets. Behaviour \& Information 30, 6: 821-831.

[64] Philip Strain, Graham McAllister, Emma Murphy, Ravi Kuber, and Wai Yu. 2007. A grid-based extension to an assistive multimodal interface. In Proceedings of Conference on Human Factors in Computing Systems: CHI'07 extended abstracts on Human factors in computing systems, 2675-2680.

[65] The Eye Tribe. 2019. The Eye Tribe website. Retrieved December 20, 2019 from https://bit.ly/36Svhfd.

[66] Tobii Dynavox. 2019. Gaze Point. Retrieved December 20, 2019 from https://www.tobiidynavox.com/en$\mathrm{gb} /$ software/free-resources/gaze-point-1/.

[67] Outi Tuisku, Veikko Surakka, Ville Rantanen, Toni Vanhala, and Jukka Lekkala. 2013. Text Entry by Gazing and Smiling. Advances in Human-Computer Interaction No. 1.

[68] Jayson Turner, Shamsi Iqbal, and Susan Dumais. 2015. Understanding gaze and scrolling strategies in text consumption tasks. In Adjunct Proceedings of the 2015 ACM International Joint Conference on Pervasive and Ubiquitous Computing and Proceedings of the 2015 ACM International Symposium on Wearable Computers, 829-838.

[69] David Ward and David MacKay. 2002. Fast hands-free writing by gaze direction. Nature 418, 6900: 838 .

[70] Andrew Wilson and Shane Williams. 2018. Autopager: exploiting change blindness for gaze-assisted reading. In Proceedings of the 2018 ACM Symposium on Eye Tracking Research \& Applications, 46.

[71] Jacob Wobbrock, James Rubinstein, Michael W. Sawyer, and Andrew T. Duchowski. 2008. Longitudinal evaluation of discrete consecutive gaze gestures for text entry. In Proceedings of the 2008 symposium on Eye tracking research \& applications, 11-18.

[72] Xiaoyu Zhao, Elias Guestrin, Dimitry Sayenko, and Tyler Simpson. 2012. Typing with eye-gaze and toothclicks. In Proceedings of the Symposium on Eye Tracking Research and Applications, 341-344.

[73]Shaojian Zhu, Yao Ma, Jinjuan Feng, and Andrew Sears. 2009. Speech-Based Navigation: Improving Grid-Based Solutions. In IFIP Conference on HumanComputer Interaction, 50-62. 